\title{
Using Nonoptimal or Archival Photographs for Constructing 3D Models
}

\author{
Robert J. Bischoff \\ (School of Human Evolution and Social Change, Arizona State University; \\ bischrob@gmail.com; corresponding author) \\ James R. Allison \\ (Department of Anthropology, Brigham Young University; jallison@,byu.edu)
}

\begin{abstract}
Photogrammetry is well on its way to becoming a standard part of archaeology. As helpful as new technology can be, it is not always applicable to past excavations. We recommend following best practices for all current work, but photographs from prior excavations are generally not ideal for photogrammetry. In this paper we demonstrate what can be done with photogrammetry under problematic conditions using photographs taken from several years to decades in the past using test cases from three archaeology sites. We discuss some of the successes, challenges, and conditions where we succeeded or failed to create a viable 3D model. Our results indicate that successful and accurate 3D models can be created from excavation photographs taken under a variety of circumstances and without accompanying camera metadata. The major limiting factor we found was lack of overlapping coverage of the subject, which proved problematic to a greater or lesser degree in all of our case studies.
\end{abstract}

Keywords: Photogrammetry, archival, 3D model 


\section{Using Nonoptimal or Archival Photographs for Constructing 3D Models}

Structure from motion $(\mathrm{SfM})$ is a branch of photogrammetry used to create 3D structure from 2D photographs. In common usage photogrammetry refers to SfM, and we follow this convention. Photogrammetry is well on its way to becoming a standard part of archaeology. As helpful as new technology can be, it is not always applicable to past excavations. We recommend following best practices for all current work, but photographs from prior excavations are generally not ideal for photogrammetry. Our purpose in this paper is not to explore the utility of photogrammetry, rather, we show what can be done with photogrammetry under problematic conditions using photographs taken from several years to decades in the past. This has been called historical, archival, or retrospective photogrammetry (Wallace 2017). We discuss some of the successes, challenges, and conditions where we succeeded or failed to create a viable 3D model. Our results indicate that successful and accurate 3D models can be created from excavation photographs taken under a variety of circumstances and without accompanying camera metadata. The major limiting factor we found was lack of overlapping coverage of the subject, which proved problematic to a greater or lesser degree in all of our case studies.

Most of the models in this study were created with Agisoft PhotoScan Standard (2018; version 1.4.1), which is widely used, inexpensive, and relatively user friendly. Thus, creating 3D models from archival or nonoptimal photographs can be done with little expense or training. Prior studies in various fields have used historical photographs with satisfactory results. Several studies used archived aerial imagery for generating photogrammetric 3D models (e.g., Bakker \& Lane 2017; Mölg \& Bolch 2017; Papworth et al. 2016; Peterson, Klein \& Steward 2015; Sevara et al. 2018), and others successfully used archival photographs for terrestrial photogrammetry (e.g., Bitelli et al. 2017; Falkingham, Bates \& Farlow 2014; Grun, Remondino \& Zhang 2004; Ioannides et al. 2013; Lallensack et al. 2015; Maiwald et al. 2017; Snavely, Seitz \& Szeliski 2008; Wallace 2017). These projects, along with our test cases discussed here, demonstrate the feasibility of creating 3D models using photographs not intended for, or optimal for, use in photogrammetry. 
We have modelled four structures at three sites in the U.S. Southwest, all in the state of Utah. We first discuss the methods used to create the 3D models and some common challenges. We then discuss the results for each 3D model we created by site. We end by summarizing our findings and recommendations.

\section{Methods}

As previously mentioned, Agisoft Photoscan was used to create the 3D models from the selected photographs. While some details are specific only to Photoscan, the general principles are likely applicable to other software, although Wallace (2017: 614) notes that Photoscan has features often better suited for what he calls "retrospective photogrammetry" than some other software. While we attempted to create one model using the professional version, all of the successful models were created using the standard version. The cost is significantly lower, which reduces the price barrier for photogrammetry. The primary benefit, in this case, for using the professional version is the ability to manually place tie points or markers on photographs to assist the software in tying photographs together (see Wallace 2017). While this can be helpful in many situations, it is encouraging that we were able to achieve satisfactory results without the added hassle of manually adding tie points, although the process often remains time consuming. This article is not a photogrammetry tutorial and is not intended to instruct a beginner, rather, we highlight the additional steps we took and challenges we encountered to achieve results using problematic photographs. While we explain some options specific to Photoscan, we will not discuss each option in detail. Our intent is to encourage others to attempt photogrammetry in similar situations and to share some methods we found helpful.

\section{Photograph selection}

The first step in photogrammetry is to acquire an image set. Generally, more photographs are better than fewer, but with some qualifications. Some difficulty is experienced when photographs were taken at different scales (i.e., close ups with long-distance overviews), but also when there are duplicate or near duplicate photographs and insufficient overlap for other photographs (see Wallace 2017: 611). In the latter case the software may only align the duplicate photographs and 
ignore other photographs. These issues can be solved by removing the problematic photographs. Our examples do not deal with photographs taken under different lighting and other conditions, but photographs taken with the same lighting, etc., is recommended. We did not have major issues using photographs that had people and objects inconsistently placed. These undesired inclusions can be masked, but we found success by merely ignoring them..

\section{Camera Alignment}

The greatest challenge we found is aligning cameras. Once accomplished, generating the 3D model is usually unproblematic, as long as a sufficient number of photographs were aligned. Camera alignment is usually automatic for good photographs, but difficulties occur when the photographs were not captured with appropriate equipment or settings. The primary cause of failed camera alignment is often insufficient coverage. Multiple camera angles are necessary for 3D construction, and the software frequently has difficulty aligning photographs taken at vastly different scales. Unfortunately, this situation usually cannot be improved in the circumstances we consider here, as the opportunity to collect more photographs is generally not an option using archival photographs.

If overlap is sufficient for a 3D model, which is often only determined after trial and error, then usually some photographs will align correctly, while others (sometimes most) of the photographs will either not be aligned or will be misaligned. The critical advice at this point in the process is to not become discouraged and to continue working with the photographs to create a proper alignment. The first step is to attempt alignment using multiple alignment configurations. What follows is a mostly nontechnical exploration of the various options related to camera alignment in Photoscan software, and which settings we recommend based on our experience and trials.

Photoscan has a number of available options, and using the highest possible settings is not always best. The primary setting for camera alignment is called "Accuracy." We generally start with high, which uses the photographs at their original scale. The highest setting upscales the photograph by 4 , while the medium setting downscales the photograph by a factor of 4 . We do not recommend lower settings than medium. Unfortunately, we cannot provide specific guidelines on when to use which setting. We find that high usually works best, but in some 
situations highest or medium settings have provided better results. "Pair preselection" can speed up the alignment process. Typically we do not select this option, but occasionally we have found better results with this option selected. We usually leave the key point tie limit at the default value, as we have found little difference when changing this value. We usually obtain best results with the "Tie point limit" set to zero, which, in this case, means there is no limit to how many points are used to tie photographs together. Using infinite tie points increases the processing time but not significantly so. Masks can be applied to remove people, objects, or scenery that is not meant to become part of the 3D model, however, we were often able to obtain good results without masking any parts of the photographs, even with people and objects variously placed within the photographs, and this step can become quite time consuming. The last option for camera alignment is "Adaptive camera model fitting," which concerns the automatic selection of camera parameters. We recommend leaving this unchecked to start, but selecting this option can sometimes improve results. The best advice we can give for these settings is to patiently experiment with your dataset and determine which settings result in the best possible camera alignment.

Often, some photographs cannot be aligned, and in the case of Figure 1 many photographs are clearly misaligned. We have found great success manually resetting camera alignments. Photoscan has several useful features to aid in this endeavor. Points that are clearly out of alignment can be selected to determine which photographs are causing the problem and can be reset and realigned. A common problem is the misalignment of axes. For example, several models we created resulted in the expected horizontal axis, while some of the photographs were misaligned resulting in one or more axes lying on different planes (see Wallace 2017: 613-614). Our recommendation is to reset all poorly aligned cameras until all aligned cameras are correctly positioned and the remaining geometry accurately represents the subject. Once accomplished the problem photographs may be realigned manually one at a time or a few at a time. If the realigned camera causes too much noise or stubbornly refuses to align properly, then it should not be used. One option to further correct this issue is to use manual tie points in the professional edition of Photoscan. But we were able to obtain good camera alignments for each model without resorting to this more time-consuming method. Figure 1 demonstrates the initial camera alignment for a 
structure discussed later. These alignment results are not promising, however, accurate geometry was captured after attempting multiple alignment configurations and manually aligning photographs. The remainder of the process typically follows standard photogrammetry procedure and does not need to be discussed here.

\section{Scale, Rotation, and Alignment}

Often, 3D models must be manually scaled to allow measurement of the model. If models must be combined, as is done in two of the case studies, then the models must not only be scaled appropriately to each other, but must also be aligned, rotated, and positioned appropriately. The open source software Blender (2018; version 2.78) was used for these purposes. Blender offers a full suite of 3D modelling tools, but has a relatively high learning curve. A full description of the processes used to scale and orient the models is beyond the scope of this paper; however, a few notes on scaling and alignment are germane. The 3D models created in Photoscan lack a true scale. An object or portion of the 3D model may have a known length, such as a scale reconstructed in the model, and this can be used to scale the entire model. Plan maps can also be used where available. As we were reconstructing architecture and the plan maps created during the original excavations were available, we found this method most effective. The basic process is to add the plan map to Blender as a background photo, scale the photograph using the scale included in the plan map, and then scale the object itself to align with the plan map. This also worked well for aligning multiple models. Figure 2 shows the plan map for both Structures 1 and 2 at Wolf Village (see case study below), which are adjacent. Figure 3 is composed of four 3D models that were aligned using the combined plan map for the structures. Blender was also used to render photographs of the $3 \mathrm{D}$ models. Photographs can be rendered with perspective or as orthophotos, which is particularly useful for archaeological applications. Automatic alignment can also be used. The open source software CloudCompare (2016; version 2.7) and Meshlab (Cignoni et al. 2008, Meshlab 2016; version 2016) both have automatic alignment options, but these options require more similarity in the 3D models than we had in our models. Determining the accuracy of the 3D models is a principal concern. The Wolf Village case study will 
demonstrate the accuracy of one of the photogrammetry 3D models compared to a 3D model created via laser scanner.

\section{Case Studies}

We have modelled four structures at three sites in the U.S. Southwest, all in the state of Utah. The sites are Wolf Village, Alkali Ridge Site 13, and Nancy Patterson. All photographs from Wolf Village and all of the photographs for the successful 3D model from Alkali Ridge Site 13 were taken using digital cameras with accompanying metadata. The photographs used in the failed reconstruction from Alkali Ridge Site 13 and the Nancy Patterson site were taken with film cameras and were scanned from negatives or from prints. While the use of scanned photographs vs digital provides different challenges for 3D modelling, several challenges were common for all of the models.

\section{Wolf Village}

The first site we created 3D models from is Wolf Village, a Native American farming village in the northern part of the state of Utah that dates primarily to the AD 1000s and early $1100 \mathrm{~s}$ (Johansson, Richards \& Allison 2014). The 3D modelling focused on two unusually large structures at the site, Structures 1 and 2. Structure 1 was a multi-room earthen surface structure. Two relatively well-constructed rooms were excavated during the 2009 and 2010 field seasons. In 2013 and 2016, approximately 20 more small rooms were excavated attached to the north side of the first two excavated. These small rooms were apparently later additions to the structure, as the walls were built on fill that rested against the north wall of the first two rooms excavated. In addition to being smaller, the walls of these rooms were thin and poorly built and floors were difficult to discern. These smaller rooms may have been used for storage, but there is little evidence that they were used at all, and one interpretation is that they were added simply to increase the size and impressiveness of the building.

Just west of the surface structure was Structure 2, a large pit structure totaling 80 square meters, including two large deep tunnels that appear to be entrances as well as a small antechamber on its southern edge. The structure was found at the end of the 2010 field season, then most of it 
was excavated in 2011 and 2012, although the southern antechamber was not excavated until 2013.

Because the excavation of these two structures was spread over six different field seasons, neither structure was ever completely uncovered at once, nor were major parts of the two adjacent structures open at the same time. At the end of the 2012 field season, when most of Structure 2 was excavated, Scott Ure completed a 3D laser scan, but the digital files were corrupted so the scan was not usable until after we completed the photo-based model we describe here.

This was fortuitous for comparative purposes, which we will discuss momentarily. Our original intent was to create a 3D model to replace the lost laser scan and to create an updated 3D model that included an antechamber excavated the year following the excavation of the main part of the structure. Additionally, we wanted to show this structure's close association with Structure 1. The structures were backfilled for preservation purposes at the end of each season, and it was impractical to remove all of the backfill each year. The creation of 3D models allowed us to combine models created from photographs taken in different years to show all completed excavations from these structures in one model.

The challenges we faced in creating these models were that these photographs were not intended for photogrammetry and were taken with a telephotographlens with different zoom levels, which is strongly not recommended for photogrammetry, and some photographs have people and various objects inconsistently moving from photograph to photo. Also, there are some gaps with too few photographs to construct a completed 3D model. A number of photographs were unusable, but we were able to create 3D models of all the desired features. This was done without masking any of the moving objects, which saved time. We combined models of Structure 2 from the 2012 excavation, the antechamber from 2013, Structure 1 as excavated in 2010, a pit in this structure that had to be modelled separately, and the remainder of Structure 1 as excavated in 2016. Of these models, only Structure 1 from 2016 was made from photographs intended for photogrammetry (these photographs were captured via an unmanned aerial vehicle). 
The alignment of the models was accomplished using the plan map of the combined structure to align the photographs within Blender. One benefit of aligning the models this way, is that we realized that the antechamber had been placed in the incorrect position on the map by almost a meter. This demonstrates an immediate benefit of these 3D models, in that they can eliminate some human error and are usually more accurate than hand-drawn maps. Figure 3 shows an orthographic render taken directly above the structures. The 3D models have a number of small holes in the models where sufficient overlap was lacking. For aesthetic purposes, these holes can be repaired using 3D modelling software, or, if 2D renders are the issue, image editor software may be used to clean the holes, rough edges, or other problems, as was done in Figure 3.

A major challenge, for this type of photogrammetry, is assessing the accuracy of the 3D model. The best method is to compare the photogrammetry model to a model collected via a laser scanner (e.g., Skarlatos \& Kiparissi 2012). Accuracy can also be compared to measurements taken directly from an artifact or feature. In the case of Structure 2 at Wolf Village, we were fortunate the lost laser scan data was recently recovered. The laser scanner is a FARO Focus 3D 120 with $\pm 2 \mathrm{~mm}$ accuracy. CloudCompare was used to compare the models. The mesh models were converted to point clouds and aligned and registered. The cloud to cloud comparison was used to determined the distance between the two point clouds. The result, Figure 4, shows the aligned point clouds and their differences. The western tunnel of this structure has the greatest variation, while many of the subsurface features are not well matched. Figure 5 shows a comparison for the west tunnel between the photogrammetry version, the laser version, and an excavation photograph used in the photogrammetry that was taken at approximately the same time as the laser scan. From these views, the photogrammetry model may best represent the physical structure of the tunnel. Photographs from the next site were taken using the same camera and the accuracy appears to be similar except for thin, stone slabs that were problematic for photogrammetry using the available photographs.

\section{Alkali Ridge Site 13}

Alkali Ridge Site 13 is an Ancestral Pueblo village in southeastern Utah that dates to the late AD 700s. As one of the earliest aggregated villages in the Four Corners area (so-called because it is 
near where the corners of the states of Utah, Colorado, New Mexico and Arizona meet), it is an important site in the archaeology of the U.S. Southwest. A large portion of the site was excavated in the 1930s (Brew 1946); our work at the site in 2012 and 2013 included re-excavating several of the rooms that had been excavated 80 years earlier, then expanding those excavations into adjacent unexcavated rooms. The impetus for creating 3D models of the excavated surface rooms at this site are similar to the reasons listed for the Wolf Village case study. Three rooms were excavated and photographed in 2012. One more complete room and portions of two others were excavated in 2013, but the 2013 excavations were not immediately adjacent and closing photographs did not overlap between these areas. We created four 3D models from excavations over these two years and combined them into one model. Compared to Wolf Village, there were fewer usable photographs, and there were a greater number of objects and people in the photographs, but the greatest difficulty was the number of relatively thin stone slabs lining the walls of the rooms. These difficulties led to several distortions in addition to the holes in the model; however, the general physical shape of the rooms turned out well and should be comparable in accuracy to the 3D models created for Wolf Village. These models were aligned by using comparable features in each model, which was later compared with an aligned plan map to verify accuracy. We found the accuracy sufficient for our purposes and, aligned together, these models allow us to render the equivalent of an aerial orthophoto (see Figure 6).

We also attempted reconstruction of some of the same rooms from photographs taken in the 1930s. While we were able to generate some 3D data, overall, we were unable to generate anything useful, even after attempting to add numerous manual tie markers in an attempt to force the software to recognize associations between photographs. While the alignment of some photographs was encouraging, we found that we lacked sufficient coverage to create a viable 3D model.

\section{Nancy Patterson}

Our last study was a reconstruction of a kiva from the Nancy Patterson Village site, another Ancestral Pueblo site in southeastern Utah. The site was used for hundreds of years, beginning in the AD 600s, but it was a large village at two different time periods: close to 900 and again in the 
1200s. The kiva we attempt to model dates to the 1200 s occupation. It was trenched in 1984 , then most of it excavated in 1985. Unfortunately, the depth of the structure, extraordinarily hard fill, the complexity of digging through and documenting the burned roof fall, and limited manpower meant that the field season ended before the floor could be completely cleared. The kiva was partially backfilled, but was still damaged by water pooling in the structure over the winter. By the start of the 1986 field season, some masonry had slumped off the wall. The excavation was finished in 1986, but we were left with photographs from 1985 that showed most of the kiva with masonry intact but the floor not completely exposed, and photographs from 1986 that showed the floor but with damaged masonry. We therefore hoped to be able to combine photographs from the two seasons to create a model that combined photographs from the two field seasons to show the structure as it would have appeared if fully excavated and undamaged.

Photographs on the project were all done with paired $35 \mathrm{~mm}$ SLR cameras, one with color film, the other with black-and-white, with the goal of producing approximately duplicated photographs with each film type. Slides were digitized using a high-resolution scanner. No metadata exists for these photographs. Our goal was to use both the black and white and color photos, thus we converted the color photographs into grayscale. Each camera was aligned separately as best as possible and then the camera calibration was saved. When both cameras were calibrated the photographs were added together and the separate calibrations were assigned to each photo. Unfortunately we were unable to align any cameras without significant distortion; however, we were able to create 3D models out of the photographs from the separate cameras. The color model as shown in Figure 7, has many holes, but the modelled geometry appears accurate. The black and white 3D model (see Figure 8) provided more coverage, but the geometry is clearly more distorted, particularly the floor of the structure. It also took many more attempts to align the cameras than for the color model. The biggest problem with this structure, is that most photographs were taken close up of separate features, and the overview photographs did not capture all sides of most parts of the kiva. We found that we lacked sufficient photographs to accomplish our purpose of merging the models to create an intact model prior to the damage between excavation seasons. 


\section{Discussion and Conclusion}

The goal of this study was to experiment with photogrammetry using photographs not explicitly meant for 3D modelling. These photographs came from both recent excavations and photographs held in archives for decades. These photographs cannot be considered optimal for photogrammetry based on technical reasons, such as a variable zoom lens, missing information, such as camera metadata, and particularly for the lack of sufficient overlap between photographs. Our purpose for these models was primarily visual, and, in some cases, we were able to obtain high quality overviews of architectural features, including the equivalent of aerial orthophotos. We found the ability to combine models made during different excavations seasons to be particularly useful. Using photogrammetry we were able to take standard excavation photos and create models showing features in contexts that did not exist during excavation. Other than lack of adequate overlap, which is difficult to rectify (although see Wallace 2017), camera alignment is the principal difficulty. This step, typically automatic, often requires time-consuming manual adjustments and experimentation with settings. Additionally, our comparison between a 3D model created from nonoptimal photographs and a precise laser scan demonstrates that these 3D models can still be accurate.

\section{Acknowledgements}

We thank Scott Ure and his assistants for their work on the laser scanning and the drone photography used in one of the 3D models. We also thank the many individuals who contributed to the excavations discussed in this paper. 
Figures

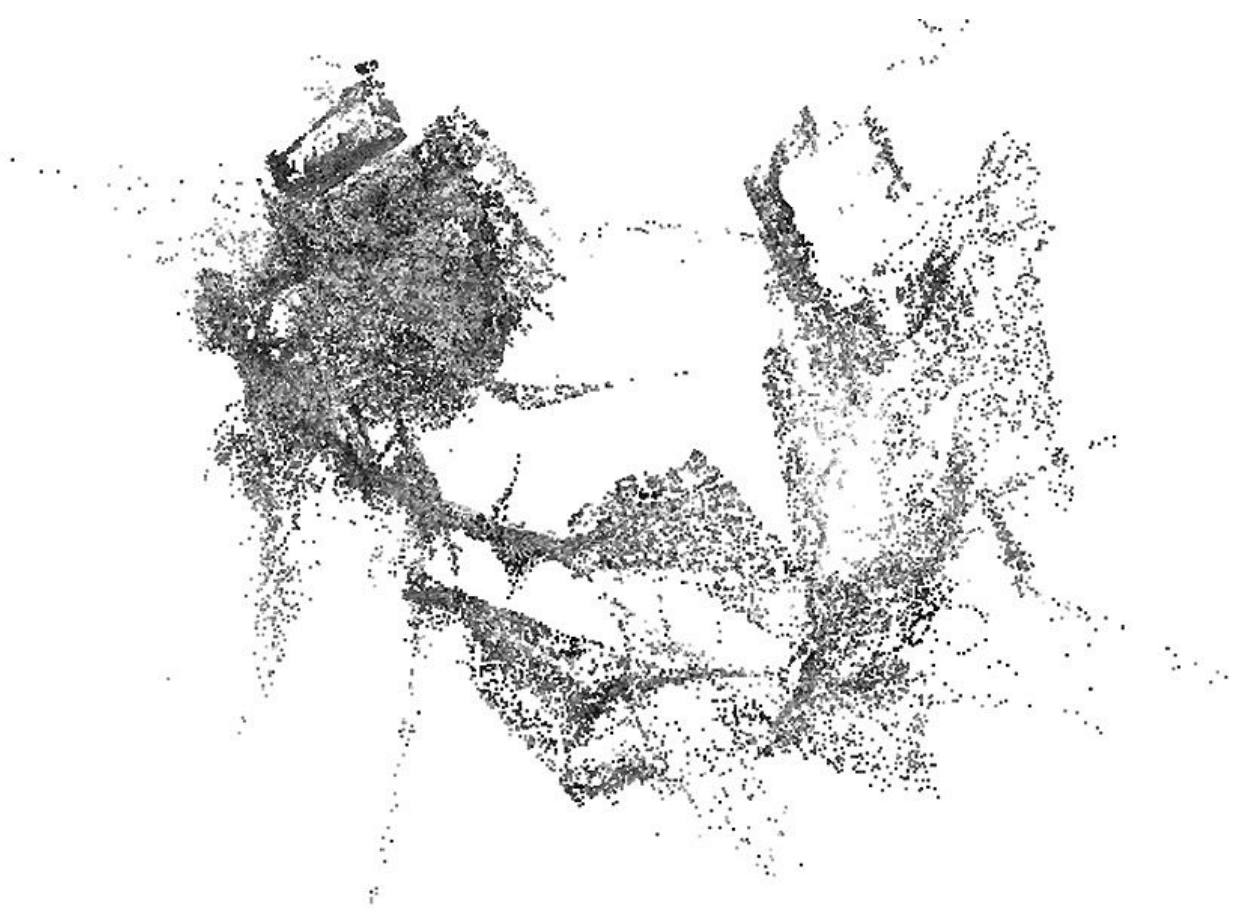

Figure 1. Demonstration of an initial, failed camera alignment in Agisoft Photoscan. 


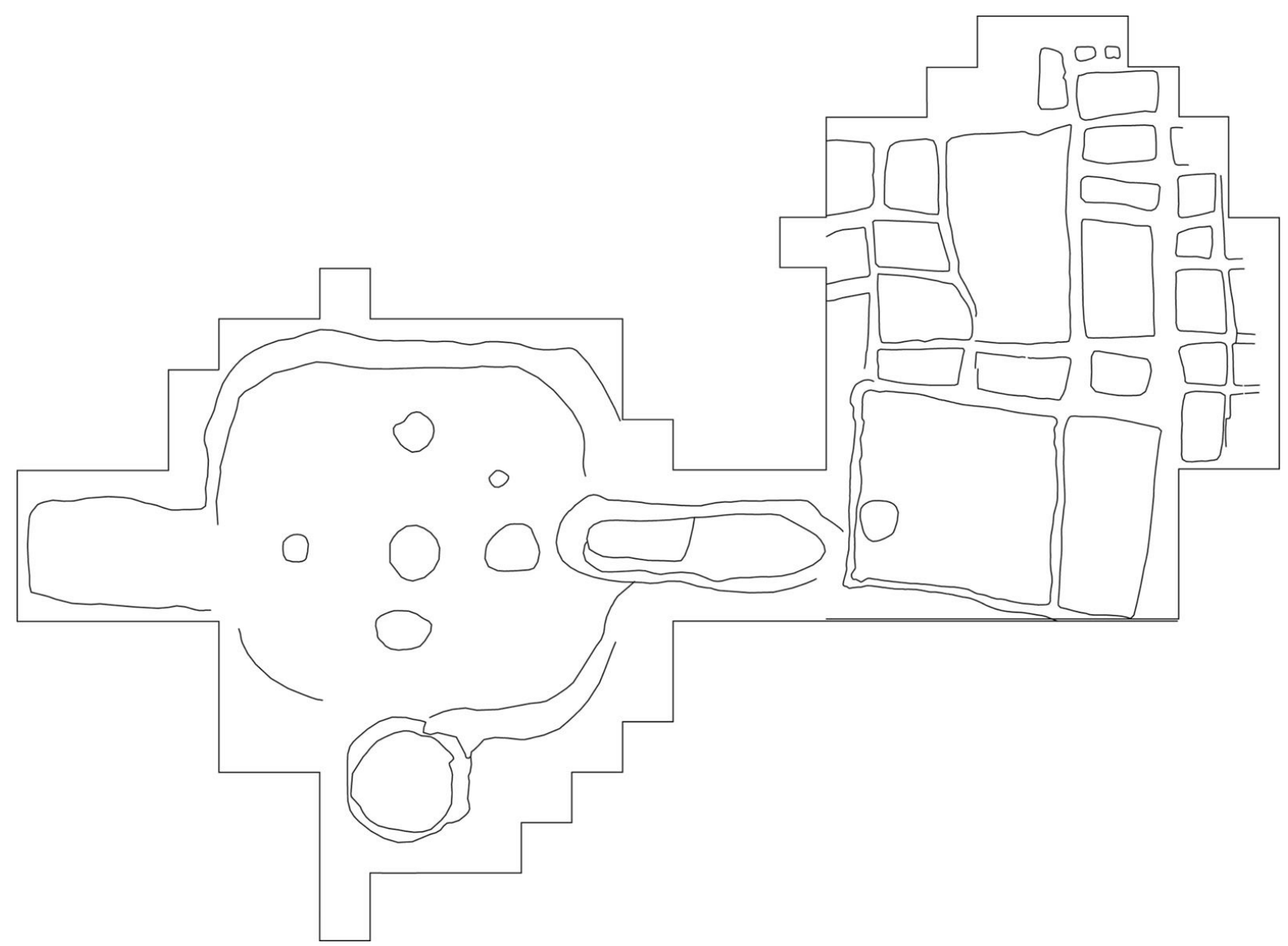

Figure 2. Plan map of Wolf Village Structures 1 and 2 used to align 3D models. 


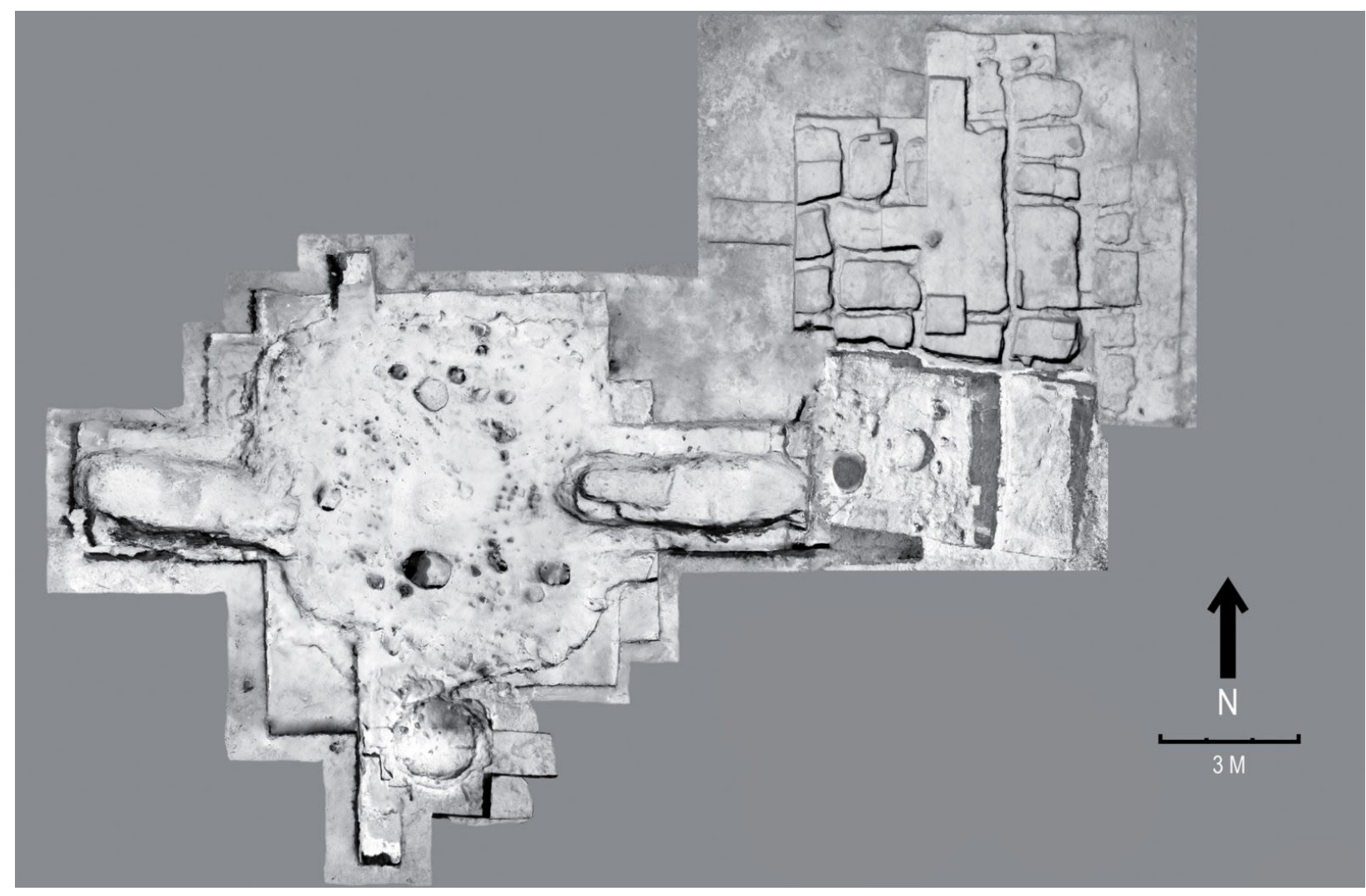


Figure 3. Render of aligned 3D models for Wolf Village Structures 1 and 2.

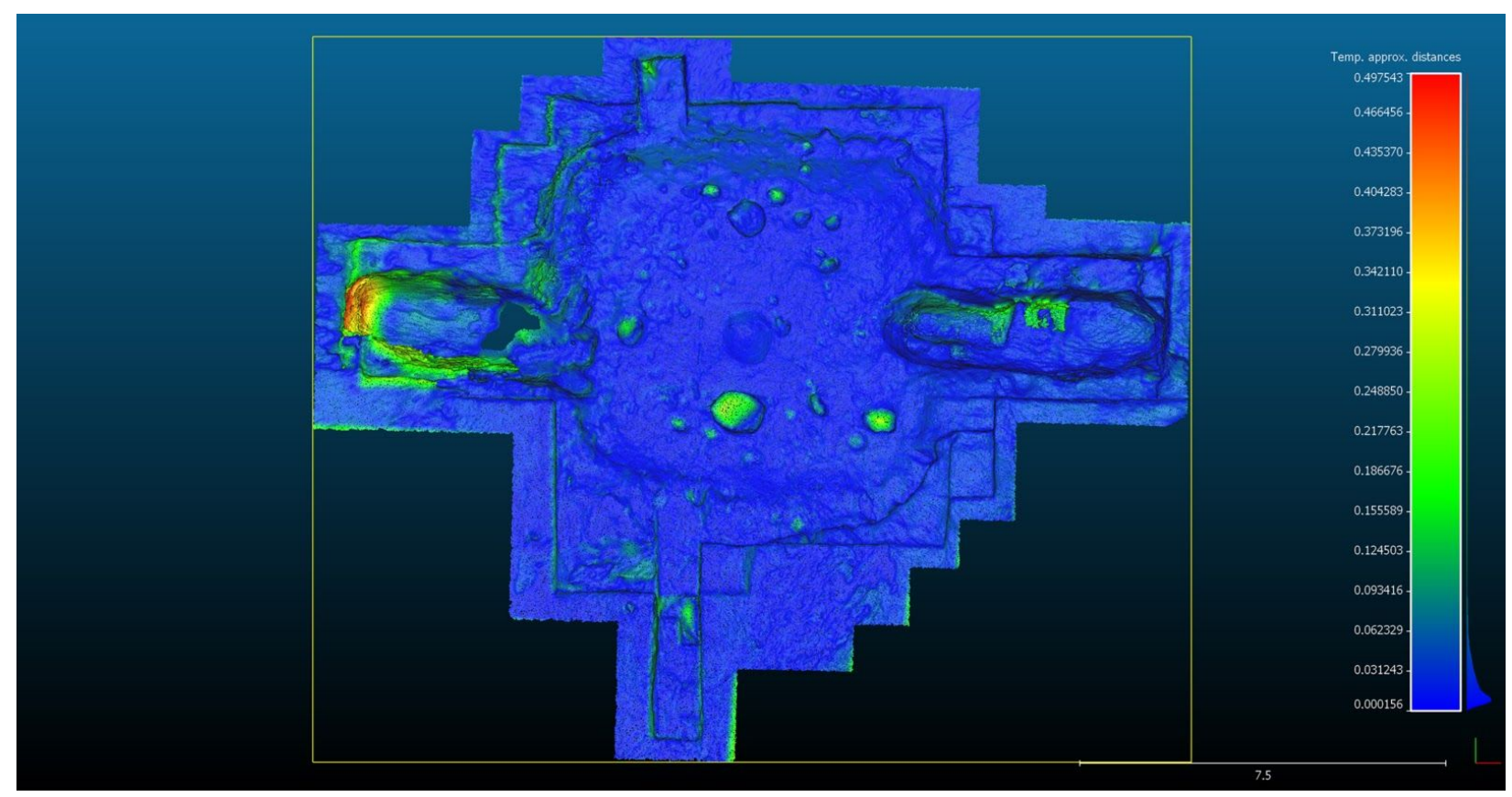

Figure 4. Cloud to cloud comparison between the laser scan and photogrammetry 3D models of Wolf Village Structure 2. 


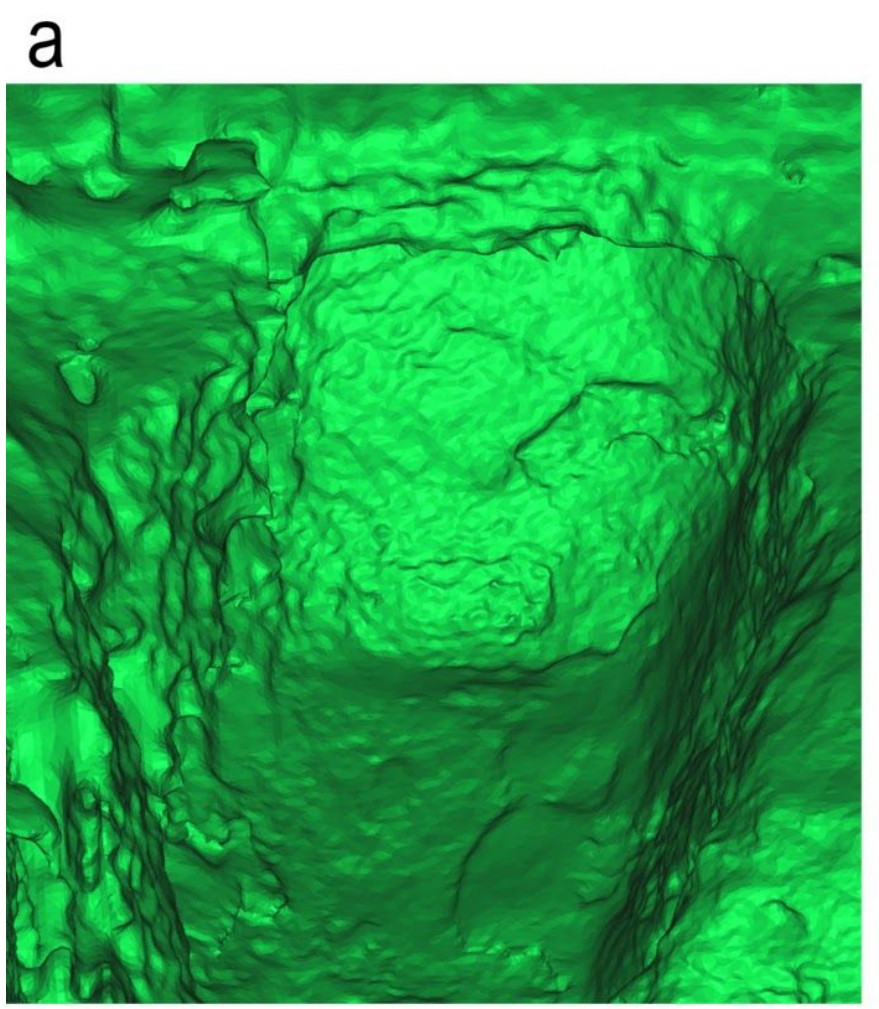

b

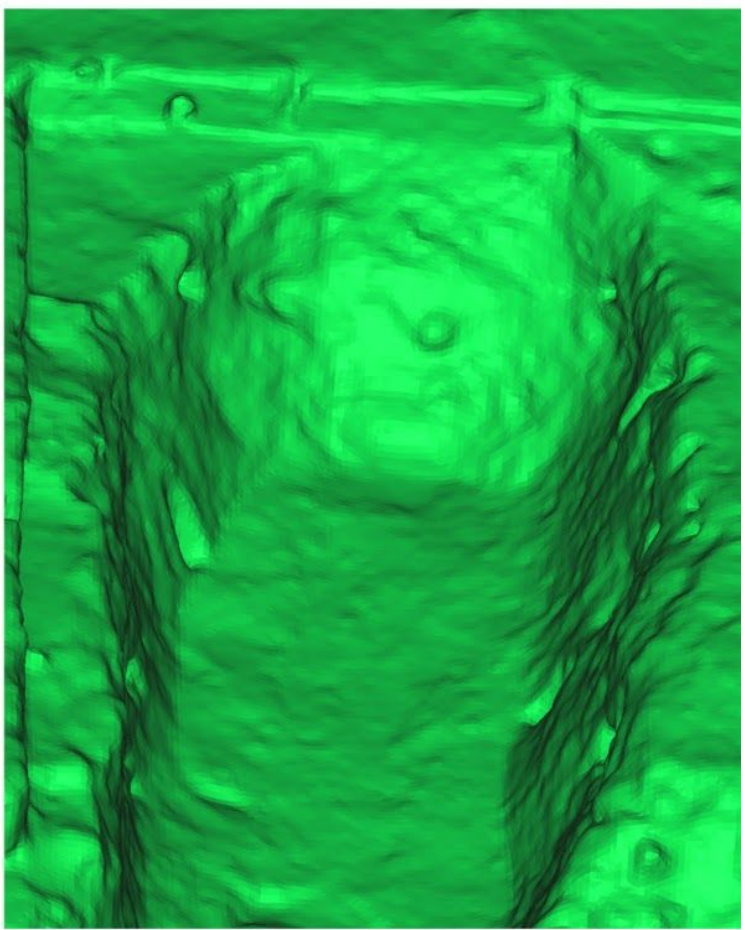

\section{C}

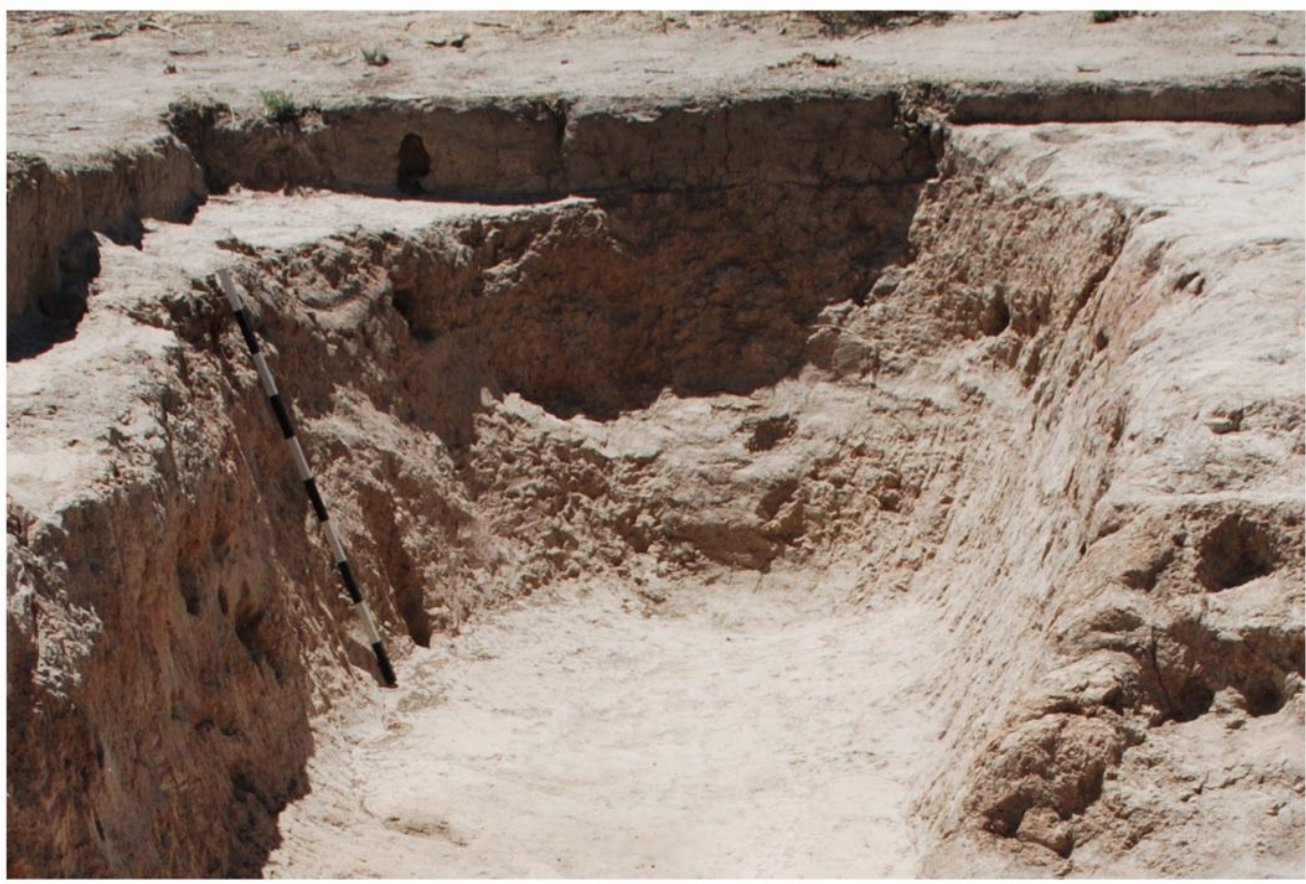

Figure 5. Comparison of the west tunnel of Wolf Village Structure 2 between the (a) photogrammetry model, (b) laser scan, and (c) excavation photo. 


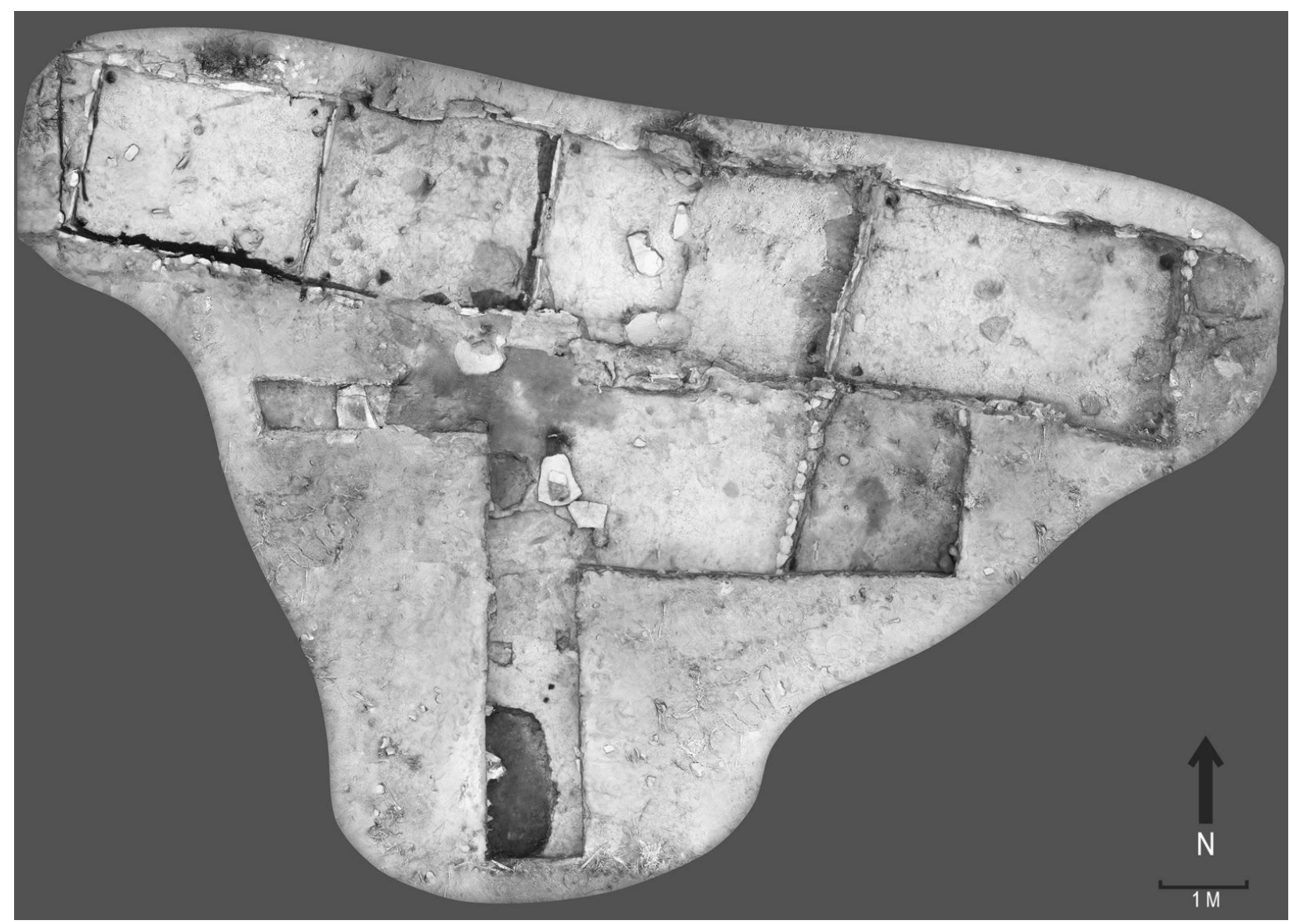

Figure 6. Render of aligned 3D models from Alkali Ridge Site 13. 


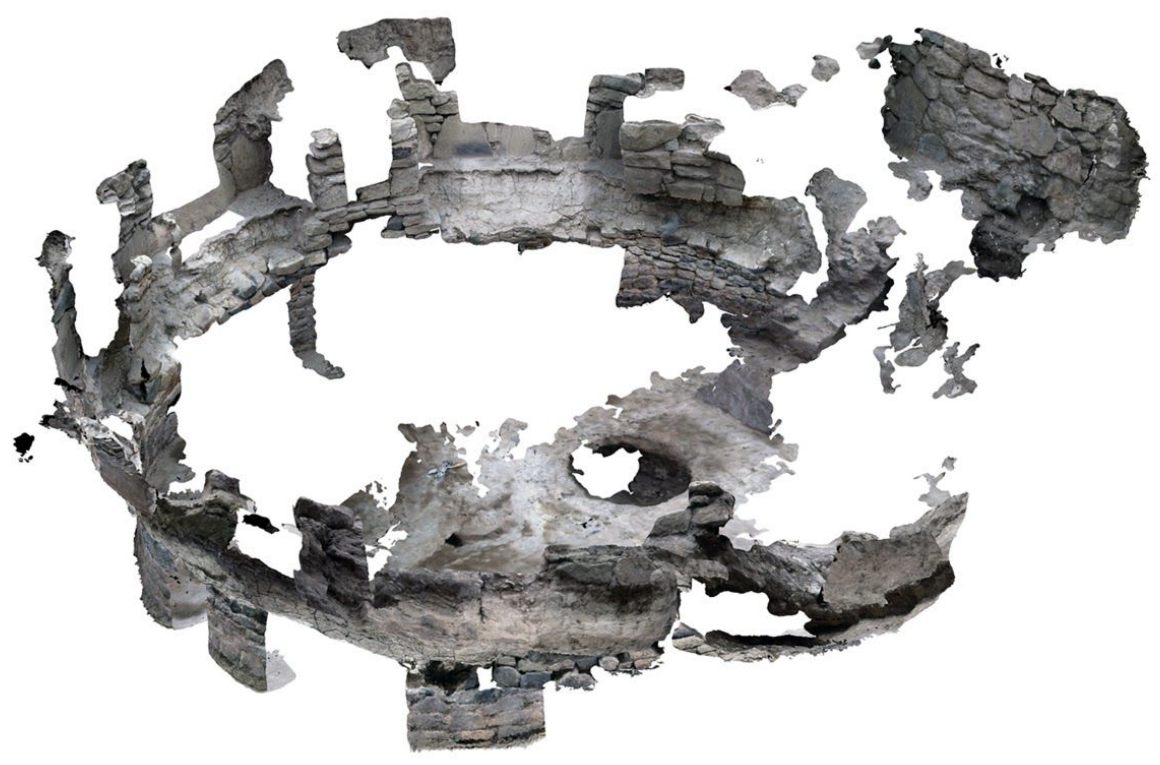

Figure 7. Render of color 3D model of Nancy Patterson kiva.

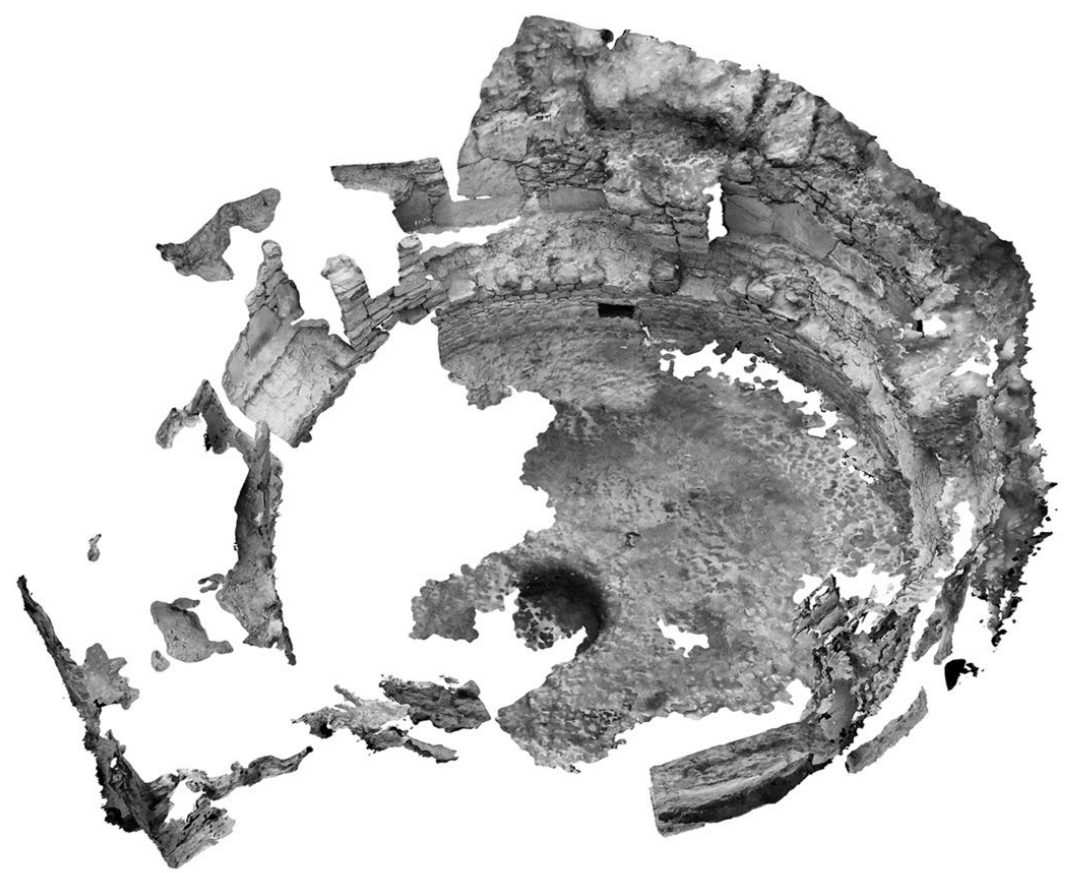

Figure 8. Render of black and white 3D model of Nancy Patterson kiva. 


\section{References}

Agisoft Photoscan Standard, computer software 2018. Available from: $<$ http://www.agisoft.com/ $>$. [last accessed 28 August 2018].

Bakker, M and Lane, S N 2017 Archival photogrammetric analysis of river-floodplain systems using Structure from Motion (SfM) methods. Earth Surface Processes and Landforms, 42(8):

1274-1286. DOI: https://doi.org/10.1002/esp.4085

Bitelli, G, Dellapasqua, M, Girelli, V, Sbaraglia, S and Tinia, M 2017 Historical photogrammetry and terrestrial laser scanning for the $3 \mathrm{~d}$ virtual reconstruction of destroyed structures: a case study in Italy. International Archives of the Photogrammetry, Remote Sensing and Spatial Information Sciences, XLII-5/W1: 113-119. DOI:

https://doi.org/10.5194/isprs-archives-XLII-5-W1-113-2017

Blender, computer software 2018. Available from: $<$ https://www.blender.org/>. [last accessed 28 August 2018].

Brew, J O 1946 Archaeology of Alkali Ridge, Southeastern Utah: With a Review of the Prehistory of the Mesa Verde Division of the San Juan and Some Observations on Archaeological Systematics. Cambridge, Massachusetts: Peabody Museum.

Cignoni, P, Callieri, M, Corsini, M, Dellepiane, M, Ganovelli, F and Ranzuglia, G 2008 MeshLab: an open-source mesh processing tool. In: Scarano, V., Chiara, R.D., and Erra, U. (eds.) Eurographics Italian Chapter Conference. The Eurographics Association. DOI: 
https://doi.org/10.2312/LocalChapterEvents/ItalChap/ItalianChapConf2008/129-136.

CloudCompare, computer software 2016. Available from: <https://www.danielgm.net/cc/>. [last accessed 28 August 2018].

Falkingham, P L, Bates, K T and Farlow, J O 2014 Historical photogrammetry: Bird's Paluxy River dinosaur chase sequence digitally reconstructed as it was prior to excavation 70 years ago. PLOS ONE, 9(4): e93247. DOI: https://doi.org/10.1371/journal.pone.0093247.

Grun, A, Remondino, F and Zhang, L 2004 Photogrammetric Reconstruction of the Great Buddha of Bamiyan, Afghanistan. The Photogrammetric Record, 19(107): 177-199. DOI: 10.1111/j.0031-868X.2004.00278.x

Ioannides, M, Hadjiprocopis, A, Doulamis, N, Doulamis, A, Protopapadakis, E, Makantasis, K, Santos, P, Fellner, D, Stork, A, Balet, O, Julien, M, Weinlinger, G, Johnson, P S, Klein, M and Fritsch, D 2013 Online 4d reconstruction using multi-images available under open access. ISPRS Annals of the Photogrammetry, Remote Sensing and Spatial Information Sciences, II-5/W1: $169-174$.

Johansson, L D, Richards, K K and Allison, J R 2014 Wolf Village (42UT273): A Case Study in Fremont Architectural Variability. Utah Archaeology, 27(1): 33-56.

Lallensack, J N, Sander, M, Knötschke, N and Wings, O 2015 Dinosaur tracks from the Langenberg Quarry (Late Jurassic, Germany) reconstructed with historical photogrammetry: Evidence for large theropods soon after insular dwarfism. Palaeontologia Electronica. DOI: 
https://doi.org/10.26879/529

Maiwald, F, Vietze, T, Schneider, D, Henze, F, Münster, S and Niebling, F 2017 Photogrammetric analysis of historical image repositories for virtual reconstruction in the field of digital humanities. ISPRS - International Archives of the Photogrammetry, Remote Sensing and Spatial Information Sciences, XLII-2/W3: 447-452. DOI:

https://doi.org/10.5194/isprs-archives-XLII-2-W3-447-2017

Meshlab, computer software 2016. Available from: <http://www.meshlab.net/>. [last accessed 28 August 2018].

Mölg, N and Bolch, T 2017 Structure-from-Motion Using Historical Aerial Images to Analyse Changes in Glacier Surface Elevation. Remote Sensing, 9(10): 1021. DOI: https://doi.org/10.3390/rs9101021

Papworth, H, Ford, A, Welham, K and Thackray, D 2016 Assessing 3D metric data of digital surface models for extracting archaeological data from archive stereo-aerial photographs. Journal of Archaeological Science, 72: 85-104. DOI: https://doi.org/10.1016/j.jas.2016.05.005

Peterson, E B, Klein, M and Steward, R L 2015 Whitepaper on structure from motion (sfm) photogrammetry: constructing three dimensional models from photography. Denver: Bureau of Reclamation.

Sevara, C, Verhoeven, G, Doneus, M and Draganits, E 2018 Surfaces from the visual past: recovering high-resolution terrain data from historic aerial imagery for multitemporal landscape analysis. Journal of Archaeological Method and Theory, 25(2): 611-642. DOI: 
https://doi.org/10.1007/s10816-017-9348-9

Skarlatos, D and Kiparissi, S 2012 Comparison of laser scanning, photogrammetry and sfm-mvs pipeline applied in structures and artificial surfaces. ISPRS Annals of Photogrammetry, Remote Sensing and Spatial Information Sciences, I-3: 299-304. DOI:

https://doi.org/10.5194/isprsannals-i-3-299-2012

Snavely, N, Seitz, S M and Szeliski, R 2008 Modeling the world from internet photo collections. International Journal of Computer Vision, 80(2): 189-210. DOI: https://doi.org/10.1007/s11263-007-0107-3

Wallace, C A B 2017 Retrospective photogrammetry in Greek archaeology. Studies in Digital Heritage, 1(2): 607-626. DOI: https://doi.org/10.14434/sdh.v1i2.23251 